\title{
APLICAÇÃO DE TÉCNICAS TRADICIONAIS E DE SÉRIES TEMPORAIS NO CONTROLE ESTATÍSTICO DA QUALIDADE
}

\author{
Maria Emilia Camargo e Helio Ricardo Friedrich \\ Departamento de Estatística - PPGMQ - Centro de Ciêncas Naturais e Exatas \\ UFSM - Santa Maria, RS
}

\section{RESUMO}

Neste trabalho é apresentado o Controle Estatístico de Qualidade da linha de produção de três máquinas da montadora de tratores MAXION, unidade de Canoas, RS, pelo Procedimento Clássico de Shewhart, bem como pelas técnicas alternativas: Somas Acumuladas (CUSUM) e Modelo de Alisamento Exponencial. Observa-se que o procedimento de Shewhart pode ser utilizado na detecção de grandes mudanças na média do processo e o Método das Somas Acumuladas é mais eficiente na detecção de pequenas mudanças na média do processo.

Palavras Chaves: Mudanças na média do processo, Controle de Qualidade, Shewhart, Somas Acumuladas, Alisamento Exponencial

\footnotetext{
ABSTRACT

In this paper the Statistical Quality Control of the production line of three machines of MAXION company, from Canoas, RS, by the basic procedure of Shewhart, as well as the alternative techniques; technique of Cumulative Sum (CUSUM) and Model of Exponential Smoothing are presented. On synthesis, notice that the procedure of Shewhart can be used at the detection of great changes at the mean of process and the method of cumulative sum is more efficient to detect little changes at the mean of process.
} 


\section{INTRODUÇÃO}

O processo de controle pode ter diferentes objetivos e consequentemente pode ser feito de diferentes formas. Considerações estatísticas associadas a processos de controle da qualidade, têm sido discutidas, por exemplo, por Aström (1970), MacGregor (1972), Hunter (1986) e Saccucci \& Lucas (1990); sobre as Somas Acumuladas encontram-se em Johnson (1961), Rendtel (1990), Reynolds et all (1990) e Radharamanan et all (1994) e aplicações de Alisamento Exponencial em controle de qualidade são apresentados em Montgomery (1968), Montgomery \& Johnson (1976), Sweet (1986) e Kirkendall (1989).

Neste artigo analisou-se os dados da empresa Maxion, através das técnicas Shewhart, Somas Acumuladas e Alisamento Exponencial, procurando identificar a constante ótima de alisamento, bem como determinando-se o número e o tempo esperado de inspeções entre a ocorrência da perturbação e a sua detecção.

O artigo divide-se em duas partes centrais, além desta introdução e da conclusão. Na sessão 2, apresenta-se a fundamentação teórica das técnicas que foram utilizadas. Na sessão 3, apresenta-se os resultados experimentais obtidos com as técnicas descritas na sessão anterior, e na sessão 4 encerra-se 0 artigo com as principais conclusões.

\section{FUNDAMENTAÇÃO TEÓRICA}

\section{1 - Método Clássico de Shewhart}

O método Clásssico de Shewhart (1931), propõe o seguinte procedimento para a construção e operação de um gráfico de controle para média de um processo caracterizado pela variável $X$, com média $\mu$ e desvio padrão $\sigma$ conhecido:

1 - A intervá.js regulares de tempo $h$, tomam-se amostras de $n$ unidades produzidas;

2 - Mede-se X para cada uma das $n$ unidades amostradas e calcula-se a média $\overline{\mathrm{X}}$; 
3 - Plota-se $\overline{\mathrm{X}}$, num gráfico seqüencial no qual estão demarcados por linhas horizontais os valores de $M$ (valor objetivo), $M-\frac{k \sigma}{\sqrt{n}}$ denominado limite inferior de controle (LIC) e $M+\frac{k \sigma}{\sqrt{n}}$, denominado limite superior de controle (LSC). Para k, Shewhart recomenda utilzar o valor 3.

4 - Enquanto os valores de $\bar{X}$ estiveram entre os limites $M \pm \frac{k \sigma}{\sqrt{n}} \circ$ processo está calibrado e em estado de controle estatístico. Caso contrário assume-se que o processo está fora de controle e deve sofrer intervenção adequada.

\section{2 - Somas Acumuladas (CUSUM)}

O método das Somas Acumuladas (CUSUM) é uma técnica bastante conhecida em Controle de Qualidade. O procedimento consiste em:

1 - A intervalos regulares de tempo $h$, tomam-se amostras de $n$ unidades produzidas, podendo ser $n=1$;

2 - Para cada amostra calcula-se a média $\bar{X}$ e para a m-ésima amostra é calculada a soma $S_{m}=\sum_{i=1}^{m}(\bar{X}-M)$, onde $M$ é o valor objetivo.

3 - Cada $S_{m}$ é plotado num gráfico, constituíndo uma máscara-V conforme a FIGURA 1. A máscara é ajustada a cada novo valor de $S_{m}$, recalculando-se $\theta$ (ângulo entre as retas horizontal e inclinada) e d (distância do vértice até o ponto $\mathrm{A}$, é chamada distância guia).

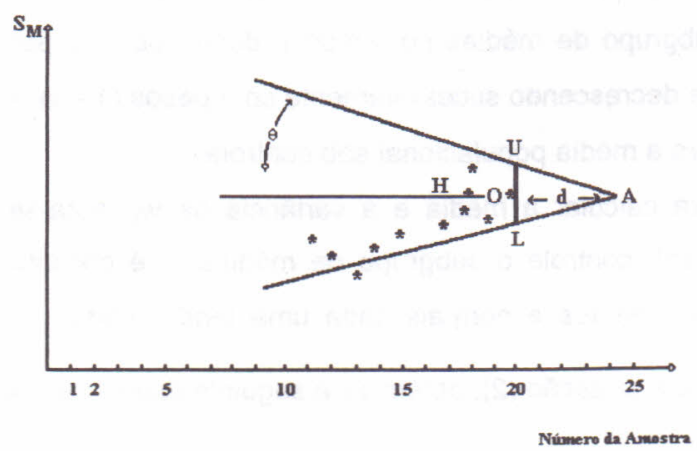

FIGURA 1 - Ilustração da Máscara - V 
4 - Se todos os pontos estiverem dentro dos braços da máscara-V, significa que a média do processo não sofreu uma alteração especificada $\delta \Sigma$, caso contrário houve perturbação no processo.

\section{3 - Modelo de Alisamento Exponencial}

Seja o modelo de alisamento exponencial simples (Montgomery \& Jonhson, 1976), dado por:

$w_{t}=\alpha \bar{x}_{t}+(1-\alpha) w_{t-1}$

onde $\alpha$ é a constante de alisamento que varia de 0 a $1, e w_{0}=\mu$.

A sequência de valores $w_{t}, t=0,1,2, \ldots$ é chamada de média móvel exponencialmente ponderada. Para entender porque é assim chamada, note que se fizermos substituições sucessivas para "w" na equação (1), obtém-se:

$$
\begin{aligned}
& \mathrm{w}_{\mathrm{t}}=\alpha \overline{\mathrm{x}}_{\mathrm{t}}+(1-\alpha)\left[\alpha \overline{\mathrm{x}}_{\mathrm{t}-1}+(1-\alpha) \mathrm{w}_{\mathrm{t}-2}\right] \\
& =\alpha \overline{\mathrm{x}}_{\mathrm{t}}+\alpha(1-\alpha) \overline{\mathrm{x}}_{\mathrm{t}-1}+(1-\alpha)^{2} \mathrm{w}_{\mathrm{t}-2} \\
& =\alpha \overline{\mathrm{x}}_{\mathrm{t}}+\alpha(1-\alpha) \overline{\mathrm{x}}_{\mathrm{t}-1}+(1-\alpha)^{2}\left[\alpha \overline{\mathrm{x}}_{\mathrm{t}-2}+(1-\alpha) \mathrm{w}_{\mathrm{t}-3}\right] \\
& =\alpha \overline{\mathrm{x}}_{\mathrm{t}}+\alpha(1-\alpha) \overline{\mathrm{x}}_{\mathrm{t}-1}+(1-\alpha)^{2} \overline{\mathrm{x}}_{\mathrm{t}-2}+\ldots+\alpha(1-\alpha)^{t-1} \overline{\mathrm{x}}_{1}+(1-\alpha)^{t} \mu,
\end{aligned}
$$

onde, pode-se observar através da equação (2) que $w_{t}$ é uma média ponderada para todo o subgrupo de médias no tempo t, dando peso $\alpha$ para os subgrupos mais recentes e decresscendo sucessivamente com pesos $(1-\alpha)$. Assim, dando-se pesos $(1-\alpha)^{t}$ para a média populacional sob controle.

Para calcular a média e a variância de $w_{\mathrm{t}}$, nota-se que, quando o processo está sob controle o subgrupo de médias $\bar{x}_{i}$ é constituído de variáveis aleatórias independentes e normais cada uma tendo média $\mu$ e variâncias $\frac{\sigma^{2}}{n}$. Portanto, usando a equação (2), obtém-se a seguinte expressåo para a média:

$$
E\left[w_{1}\right]=\mu\left[\alpha+\alpha(1-\alpha)+\alpha(1-\alpha)^{2}+\ldots+\alpha(1-\alpha)^{t-1}+(1-\alpha)^{t}\right]
$$




$$
=\frac{\mu \alpha\left[1-(1-\alpha)^{t}\right]}{1-(1-\alpha)}+\mu(1-\alpha)^{t}
$$

Para "t" grande, e quando o processo esta sob controle, temos que:

$\mathrm{E}\left[\mathrm{w}_{\mathrm{t}}\right]=\mu$.

A variância é dada por:

$$
\begin{aligned}
\operatorname{Var}\left[w_{t}\right] & =\frac{\sigma^{2}}{n}\left\{\alpha^{2}+[\alpha(1-\alpha)]^{2}+\left[\alpha(1-\alpha)^{2}\right]^{2}+\ldots+\left[\alpha(1-\alpha)^{t-1}\right]^{2}\right\} \\
& \text { Fazendo } \beta=(1-\alpha)^{2}, \text { temos: } \\
\operatorname{Var}\left[w_{t}\right] & =\frac{\sigma^{2}}{n} \alpha^{2}\left[1+\beta+\beta^{2}+\ldots+\beta^{t-1}\right] \\
& =\frac{\sigma^{2} \alpha^{2}\left[1-(1-\alpha)^{2 t}\right]}{n\left[1-(1-\alpha)^{2}\right]} \\
& =\frac{\sigma^{2} \alpha\left[1-(1-\alpha)^{2 t}\right]}{n(2-\alpha)} .
\end{aligned}
$$

\section{APRESENTAÇÃO E ANÁLISE DOS RESULTADOS}

Os dados analisados nesse trabalho foram coletados na unidade da Empresa MAXION, estabelecida na cidade de Canoas - RS, fabricante dos tratores Maxion. Tais dados são referentes à produção de três máquinas, aqui identificadas pelos seus números, as quais fabricam três peças, que constavam do programa de Controle Estatístico de Qualidade da empresa. Para efeito de controle foram colhidas amostras de 5 unidades produzidas, a cada hora, durante um dia de trabalho.

Em cada caso, o valor objetivo ou a média é o valor nominal da dimensão controlada e o desvio-padrão $\sigma$ é conhecido. O conhecimento de $\sigma$ justifica-se pelo fato de ter sido obtido de uma amostra de 1000 observações de cada máquina no período de 4 de março a 13 de abril de 1993.

Os resultados serão apresentados em função da constante ótima de alisamento, do Número Esperado $E(N)$ e do Tempo Esperado $E(T)$ para as três 
máquinas, bem como a verificação da Eficiência Relativa (ER) entre os procedimentos de Shewart, Somas Acumuladas e Alisamento Exponencial.

\section{1 - Verificação da constante de alisamento}

Na TABELA 1 apresentam-se os valores de $\alpha$ para as três máquinas, bem como as variâncias residuais com o objetivo de determinar a constante ótima.

TABELA 1 - Variância residual para as três máquinas conforme a variação da constante de alisamento

\begin{tabular}{c|c|c|c|c|c}
\hline \hline \multicolumn{2}{c|}{ MÁQUINA 4401 } & \multicolumn{2}{c|}{ MÁQUINA 2615 } & \multicolumn{2}{c}{ MÁQUINA 4232 } \\
\hline $\begin{array}{c}\text { Cte. de } \\
\text { Alisamento }(\alpha)\end{array}$ & $\begin{array}{c}\text { Variância } \\
\text { Residual }\end{array}$ & $\begin{array}{c}\text { Cte. de } \\
\text { Alisamento } \\
\end{array}$ & $\begin{array}{c}\text { Variância } \\
\text { Residual }\end{array}$ & $\begin{array}{c}\text { Cte. de } \\
\text { Alisamento } \\
(\alpha)\end{array}$ & $\begin{array}{c}\text { Variância } \\
\text { Residual }\end{array}$ \\
\hline \multirow{2}{*}{0,1} & $0,19199 \mathrm{E}-06$ & 0,1 & $0,42846 \mathrm{E}-02$ & $* 0,1$ & $0,42890 \mathrm{E}-07$ \\
0,5 & $0,22233 \mathrm{E}-06$ & $* 0,5$ & $0,42807 \mathrm{E}-02$ & 0,5 & $0,46206 \mathrm{E}-07$ \\
0,7 & $0,20203 \mathrm{E}-06$ & 0,7 & $0,42818 \mathrm{E}-02$ & 0,7 & $0,47055 \mathrm{E}-07$ \\
0,9 & $0,21560 \mathrm{E}-06$ & 0,9 & $0,42812 \mathrm{E}-02$ & 0,9 & $0,51175 \mathrm{E}-07$ \\
\hline
\end{tabular}

As constantes assinaladas são as constantes de alisamento ótimas sendo que para as máquinas 4401 e 4232, $\alpha=0.1$, e para a máquina $2615 \alpha=0.5$.

\section{2 - Determinação do Número Esperado $E(N)$ e Tempo Esperado $E(T)$}

A seguir, apresenta-se, $p_{1}$, a probabilidade de alarme em cad inspeção, o Número Esperado de inspeções entre a ocorrência de ur perturbação e sua detecção, bem como o Tempo médio decorrido entre ocorrência e a detecção de uma perturbação.

Na TABELA 2, apresentam-se os valores de $p_{1}, E\left(N_{1}\right)$ e $E\left(T_{1}\right)$ pa diversos valores de $\delta$ (magnitude da perturbação estável), o número de desvio padrões, para o procedimento de Shewhart. 
TABELA 2 - Representação de $p_{1}, E\left(N_{1}\right)$ e $E\left(T_{1}\right)$ para diversos valores de $\delta$ Procedimento de Shewhart

\begin{tabular}{c|c|c|c}
\hline \hline$\delta$ & $p_{1}$ & $E\left(N_{1}\right)$ & $E\left(T_{1}\right)$ \\
\hline 0 & 0.0027 & 370 & 370 \\
0.25 & 0,0075 & 132,80 & 132,30 \\
0.5 & 0.0301 & 33,26 & 32,76 \\
1.0 & 0.2236 & 4,47 & 3,97 \\
1.5 & 0.6368 & 1,57 & 1,07 \\
2.0 & 0.9292 & 1,08 & 0,58 \\
3.0 & 0.9999 & 1,00 & 0,50 \\
4.0 & 1,0000 & 1,00 & 0,50 \\
\hline
\end{tabular}

$\mathrm{Na}$ TABELA 3 apresenta-se o comportamento de $E\left(\mathrm{~N}_{2}\right)$ e $E\left(\mathrm{~T}_{2}\right)$ fazendo-se $0 \delta$ variar de 0 a 4, para o procedimento das Somas Acumuladas.

TABELA 3 - Valores de $\mathrm{p}_{1}$, $\mathrm{E}\left(\mathrm{N}_{2}\right)$ e $\mathrm{E}\left(\mathrm{T}_{2}\right)$ para diversos valores de $\delta$ Procedimento de Somas Acumuladas

\begin{tabular}{c|c|c|c}
\hline \hline$\delta$ & $\mathbf{p}_{1}$ & $\mathbf{E}\left(\mathrm{N}_{2}\right)$ & $\mathbf{E}\left(\mathrm{T}_{2}\right)$ \\
\hline 0.0 & 0,0027 & 370 & 370 \\
0.25 & 0,0131 & 76,5 & 76,3 \\
0.50 & 0,0391 & 25,6 & 25,1 \\
1.00 & 0,1010 & 9,90 & 9,4 \\
1.50 & 0,1639 & 6,10 & 5,6 \\
2.00 & 0,2222 & 4,50 & 4,0 \\
3.00 & 0,3333 & 3,0 & 2,5 \\
4.00 & 0,4348 & 2,3 & 1,8 \\
\hline
\end{tabular}

Nas TABELAS 4 e 5 apresentam-se os valores de $p_{1}, E\left(N_{3}\right)$ e $E\left(T_{3}\right)$ para os diversos valores de $\delta$, considerando $\alpha=0.1$ e $\alpha=0.5$, respectivamente, para o procedimento de Alisamento Exponencial. 
TABELA 4 - Representação de $p_{1}, E\left(N_{3}\right)$ e $E\left(T_{3}\right)$ para diversos valores de $\delta$. Máquinas 4401 e 4232 - Procedimento de Alisamento Exponencial

\begin{tabular}{c|c|c|c}
\hline \hline$\delta$ & $\mathbf{p}_{1}$ & $\mathbf{E}\left(\mathbf{N}_{\mathbf{3}}\right)$ & $\mathbf{E}\left(\mathbf{T}_{\mathbf{3}}\right)$ \\
\hline 0 & 0,0027 & 370 & 370 \\
0.25 & 0,0212 & 47,17 & 46,67 \\
0.5 & 0,1446 & 6,92 & 6,42 \\
1.0 & 0,8078 & 1,24 & 0,74 \\
1.5 & 0,9976 & 1,00 & 0,50 \\
2.0 & 1,0000 & 1,00 & 0,5 \\
2.5 & 1,0000 & 1,00 & 0,5 \\
3.0 & 1,0000 & 1,00 & 0,5 \\
4.0 & 1,0000 & 1,00 & 0,5 \\
\hline
\end{tabular}

TABELA 5 - Representação de $p_{1}, E\left(N_{3}\right)$ e $E\left(T_{3}\right)$ para diversos valores de $\delta$. Máquina 2615

\begin{tabular}{c|c|c|c}
\hline \hline$\delta$ & $\mathbf{p}_{1}$ & $\mathbf{E}\left(\mathbf{N}_{3}\right)$ & $\mathbf{E}\left(\mathrm{T}_{3}\right)$ \\
\hline 0 & 0,0027 & 370 & 370 \\
0.25 & 0,0088 & 113,51 & 280,40 \\
0.5 & 0,0392 & 25,51 & 25,01 \\
1.0 & 0,2981 & 3,36 & 2,86 \\
1.5 & 0,7612 & 1,31 & 0,81 \\
2.0 & 0,9738 & 1,03 & 0,53 \\
2.5 & 0,9992 & 1,00 & 0,50 \\
3.0 & 1,0000 & 1,00 & 0,50 \\
4.0 & 1,0000 & 1,00 & 0,50 \\
\hline
\end{tabular}

\section{3 - Verificação da Eficiência Relativa entre os procedimentos}

A eficiência relativa de uma técnica em relação a outra, é a relação entre o tempo esperado de inspeções, desde a ocorrência até a detecção da perturbação, de uma técnica considerada padrão pela outra considerada alternativa. Quanto maior a freqüência relativa, maior a eficiência da técnica alternativa. 
Nas TABELAS 6 e 7 apresentam-se a Eficiência Relativa para o Número Esperado de inspeçőes e o Tempo Médio decorrido entre a ocorrência de uma perturbação e sua detecção.

TABELA 6 - Valores de $E\left(N_{1}\right)$ para o procedimento de Shewhart, $E\left(N_{2}\right)$ para o procedimento CUSUM, $\mathrm{E}\left(\mathrm{N}_{3}\right)$ para o Alisamento Exponencial e da Eficiência Relativa

\begin{tabular}{c|c|c|c|c|c|c}
\hline \hline$\delta$ & $E\left(\mathbf{N}_{\mathbf{1}}\right)$ & $E\left(\mathbf{N}_{\mathbf{2}}\right)$ & $E\left(\mathbf{N}_{\mathbf{3}}\right)$ & $\begin{array}{c}E\left(\mathbf{N}_{\mathbf{1}}\right) / E\left(\mathbf{N}_{\mathbf{2}}\right. \\
)\end{array}$ & $\begin{array}{c}E\left(\mathbf{N}_{\mathbf{2}}\right) / E(\mathbf{N} \\
\mathbf{3})\end{array}$ & $\begin{array}{c}E\left(\mathbf{N}_{\mathbf{1}}\right) / E(\mathbf{N} \\
3)\end{array}$ \\
\hline 0.0 & 370 & 370 & 370 & 1,00 & 1,00 & 1,00 \\
0.25 & 132,80 & 76,50 & 47,17 & 1,74 & 1,62 & 2,82 \\
0.50 & 33,26 & 25,60 & 6,92 & 1,30 & 3,70 & 4,81 \\
1.00 & 4,47 & 9,90 & 1,24 & 0,45 & 7,98 & 3,60 \\
1.50 & 1,57 & 6,10 & 1,00 & 0,26 & 6,10 & 1,57 \\
2.00 & 1,08 & 4,50 & 1,00 & 0,24 & 4,50 & 1,08 \\
3.00 & 1,00 & 3,00 & 1,00 & 0,33 & 3,00 & 1,00 \\
4.00 & 1,00 & 2,30 & 1,00 & 0,43 & 2,30 & 1,00 \\
\hline
\end{tabular}

TABELA 7 - Valores de $E\left(T_{1}\right)$ para o procedimento Shewhart, $E\left(T_{2}\right)$ para o procedimento CUSUM, $E\left(T_{3}\right)$ para o Alisamento Exponencial e da Eficiência Relativa

\begin{tabular}{c|c|c|c|c|c|c}
\hline \hline$\delta$ & $E\left(T_{1}\right)$ & $E\left(T_{2}\right)$ & $E\left(T_{3}\right)$ & $E\left(T_{1}\right) / E\left(T_{2}\right)$ & $E\left(T_{2}\right) / E\left(T_{3}\right)$ & $E\left(T_{1}\right) / E\left(T_{3}\right)$ \\
\hline 0.0 & 370 & 370 & 370 & 1,00 & 1,00 & 1,00 \\
0.25 & 132,30 & 76,3 & 46,67 & 1,73 & 1,63 & 2,83 \\
0.50 & 32,76 & 25,1 & 6,42 & 1,31 & 3,91 & 5,10 \\
1.00 & 3,97 & 9,4 & 0,74 & 0,42 & 12,70 & 5,36 \\
1.50 & 1,07 & 5,6 & 0,50 & 0,19 & 11,20 & 2,14 \\
2.00 & 0,58 & 4,0 & 0,50 & 0,15 & 8,00 & 1,16 \\
3.00 & 0,50 & 2,5 & 0,50 & 0,20 & 5,00 & 1,00 \\
4.00 & 0,50 & 1,8 & 0,50 & 0,28 & 3,60 & 1,00 \\
\hline
\end{tabular}

Observando-se as TABELAS 6 e 7, pode-se dizer que o modelo da somas acumuladas é mais eficiente que o modelo de Shewhart na deteç̧ão $d$. pequenas perturbações $(\delta=0,25$ e $\delta=0,5)$ na média ou valor objetivo do 
processo, visto que sua Eficiência Relativa é maior que $1(1,73$ e 1,3') e o Modelo de Alisamento Exponencial, é mais eficiente que o CUSUM para perturbações da ordem de $\delta=0$ a $\delta=3,0$.

O procedimento de Shewhart é mais eficiente que o método CUSUM na detecção de grandes mudanças, da ordem de $\delta=1$ a $\delta=4$, enquanto que 0 método de Alisamento Exponencial é mais eficiente nos demais casos.

\section{CONCLUSÃO}

O presente trabalho encontrou base nas técnicas tradicionais de Controle Estatístico de Qualidade, bem como nas técnicas alternativas das Somas Acumuladas e Modelos de Alisamento Exponencial.

A tecnica de Shewhart pode ser utilizada no controle de processos nos quais haja grandes mudanças na média, devido à lentidão com que acusa alarmes falsos. Já o método das Somas Acumuladas é mais eficiente na detecção de pequenas mudanças na média. O Modelo de Alisamento Exponencial, inclui as duas técnicas, como casos limites, pois quando $\alpha=10$ alisamento exponencial equivale à técnica de Shewhart, e quando $\alpha=0$ equivale à técnica das Somas Acumuladas.

\section{5 - REFERÊNCIAS BIBLIOGRÁFICAS}

ASTRÖM, K.J.(1970). Introduction to stochastic control. Mathematics in science and engeneering series. 70. Academic Press.

HUNTER, J. S. (1986). The exponentially weighted moving average. Journal of Quality Technology 18, p. 203-210.

JOHNSON, N. L. (1961). A simple theoretical approach to cumulative sum control chart. Journal of the American Statistical Association, 56, p. 835-840.

KIRKENDALL, N. J. (1989). The relationship between Certain Kalman Filter Models and Exponential Smoothing Models. Quality and reability. 
MAcGREGOR, J. F. (1972). Topics in the control of linear processes subject to stochastic disturbances. Ph.D. dissertation, University of Wisconsin Madison.

MONTGOMERY, D. C. (1968). Introduction to statistical Quality Control. Wiley, New York.

MONTGOMERY, D. C. \& JOHNSON, L. A. (1976). Forecasting and time series analysis. New York, McGraw-Hill.

RADHARAMANAN, R.; GALELLI, D. T. \& PERES, L. L. (1994). Sensitivity analysis on the CUSUM method Amsterdan, Elsevier, International Journal of Production Economics, v. 33, p. 89 - 95.

REYNOLDS, M. R. Jr; AMIM, R. W. \& ARNOLD, J. C. (1990). CUSUM chart wich variable sampling intervals. Technometrics, vol 32, p. 371 - 384.

SACCUCCI, M. S. \& LUCAS, J. M. (1990). Average run lengths for exponnencially weighted moving average control schemes using the Markov chain approach. Jornal of Quality Technology, vol. 22, p. 154 - 162.

SHEWHART, W. A. (1931) . Economic control of Quality of manufactured product. New York, D. Van Nostrand.

SWEET, A. (1986). Control charts using compled exponencially weighted moving averages. II E Transaction, March, 26 - 33. 
